\title{
Fertility preservation for women with ovarian endometriosis: it is time to adopt it as routine practice FOR: Fertility preservation is an integral component of the management of women with ovarian endometriosis
}

\author{
Sania Latif ${ }^{1}$, Ertan Saridogan ${ }^{1}$, and Ephia Yasmin ${ }^{1}$ \\ ${ }^{1}$ University College London Hospitals NHS Foundation Trust
}

August 24, 2021

Fertility preservation for women with ovarian endometriosis: it is time to adopt it as routine practice

FOR: Fertility preservation is an integral component of the management of women with ovarian endometriosis

Sania Latif ${ }^{1}$, Ertan Saridogan ${ }^{1}$, Ephia Yasmin ${ }^{1}$

${ }^{1}$ Reproductive Medicine Unit, University College London Hospital NHS FoundationTrust

\section{Corresponding author:}

Miss Ephia Yasmin ephia.yasmin@nhs.net

Reproductive Medicine Unit

University College London Hospital

235 Euston Road

London NW1 2BU

Keywords: fertility preservation, oocyte cryopreservation, embryo cryopreservation, endometriosis, infertility

FOR: Fertility preservation is an integral component of the management of women with ovarian endometriosis

Fertility preservation techniques are widely accepted as standard of care for women undergoing treatment for cancer who are at risk of premature ovarian insufficiency. The same approach is not yet well established in benign conditions.

There is ample evidence that women who have endometriosis are twice as likely to experience infertility in the future (Prescott et al. 2016Hum Reprod 31(7):1475-82). Ovarian endometriomas reduce ovarian reserve by exposing healthy ovarian tissue to the pathological process of endometriosis and to mechanical stretch, resulting in a progressive reduction in the pool of primordial follicles. Surgery to treat ovarian endometriomas further reduces ovarian reserve due to loss of normal ovarian tissue during cystectomy and ablation. Following surgical removal, there is a reduction in ovarian reserve, as measured by anti-mullerian hormone $(\mathrm{AMH})$ levels, by $30 \%$ in unilateral and $44 \%$ in bilateral endometriomas (Raffi et al. 2012 , $J$ Clin Endocrinol Metab. 97:3146-3154). The risk of premature ovarian insufficiency after bilateral ovarian 
endometrioma removal is 2.4\% (Busacca et al. 2006, Am J Obstet Gynecol 195(2):421-5).Younger women have a higher recurrence rate of endometriomas requiring repeated surgery, which compounds the insult to their ovarian reserve. Accepting this risk, the European Society for Gynaecological Endoscopy, the European Society for Human Reproduction and Embryology and the World Endometriosis Society have collaborated in developing recommendations on the practical aspects of endometrioma surgery to reduce their adverse impact. Women with endometriosis are often subjected to the pressure of early childbearing based on their risk of infertility, whilst there is a societal trend towards delaying parenthood.

Success rates of IVF are dependent on oocyte yield. The number of oocytes retrieved from women with endometriomas undergoing ovarian stimulation is substantially reduced, particularly in the presence of large and bilateral endometriomas (Kim et al. 2020, RBMO40(6):827-834). It is, however, possible to restore cumulative livebirth rates in women with endometriosis when an equivalent number of oocytes is retrieved (Cobo et al. 2021, RBMO 42:725-732). There is evidence that almost half of women who had oocyte cryopreservation due to endometriosis subsequently used their oocytes, highlighting substantial utilisation of stored gametes within this group of women (Cobo et al. 2020, Fertility and Sterility 113:836-844).

In light of this information, it is difficult to justify excluding women with endometriosis from having fertility preservation. A structured approach is required to grade the risk to fertility in endometriosis rather than questioning the validity of fertility preservation in these women. In order to construct criteria for offering fertility preservation, prospective data collection is required to understand long-term fertility patterns. Size of endometrioma, bilaterality, previous surgery and age are the obvious candidates in determining risk.

An early discussion around reproductive planning is essential in women with ovarian endometriosis regarding the implications of their significant risk of diminished ovarian reserve and premature ovarian insufficiency. Oocyte and embryo cryopreservation offer women with ovarian endometriosis an effective and reliable option to increase their chance of reproductive success, particularly in young women with large or bilateral endometriomas who may require surgical intervention, those who have had previous surgery and those who are not in a position to embark upon pregnancy. It is imperative that the risk to ovarian reserve and fertility as well as the role of fertility preservation are recognised in women with ovarian endometriosis, just as in women undergoing treatment for malignancies.

Word count 500 (excluding references)

\section{References}

1. Prescott J, Farland LV, Tobias DK, Gaskins AJ, Spiegelman D, Chavarro JE, Rich-Edwards JW, Barbieri RL, Missmer SA. A prospective cohort study of endometriosis and subsequent risk of infertility. Hum Reprod. 2016 Jul;31(7):1475-82. doi: 10.1093/humrep/dew085. Epub 2016 May 1. PMID: 27141041; PMCID: PMC4901880.

2. Raffi F, Metwally M, Amer S. The impact of excision of ovarian endometrioma on ovarian reserve: a systematic review and meta-analysis. J Clin Endocrinol Metab. 2012; 97: 3146-3154 https://doi.org/10.1016/j.fertnstert.2018.06.025.

3. Busacca M, Riparini J, Somigliana E, Oggioni G, Izzo S, Vignali M, Candiani M. Postsurgical ovarian failure after laparoscopic excision of bilateral endometriomas. Am J Obstet Gynecol. 2006 Aug;195(2):421-5. doi: 10.1016/j.ajog.2006.03.064.

4. Kim SK, Kim SJ, Lee J, Suh C, Kim S. Oocyte cryopreservation for fertility preservation in women with ovarian endometriosis. Reproductive BioMedicine Online 2020 40(6) 827-834.

5. Cobo A, Coello A, José de los Santos M, Giles J, Pellicer A, Remohí J, García-Velasco J. Number needed to freeze: cumulative live birth rate after fertility preservation in women with endometriosis. Reproductive BioMedicine Online 2021 42: 725-732. https://doi.org/10.1016/j.rbmo.2020.12.013.

6. Cobo A, Giles J, Paolelli S, Pellicer A, Remohí J, García-Velasco J. Oocyte vitrification for fertility preservation in women with endometriosis: an observational study. Fertility and Sterility 2020113 : 836-844. https://doi.org/10.1016/j.fertnstert.2019.11.017.

\section{Funding}


This research received no specific grant from any funding agency in the public, commercial or not-for-profit sectors.

\section{Author Statement}

SL, ES and EY drafted, revised and approved the final manuscript and are in agreement to be accountable for all aspects of the work.

\section{Conflict of Interests}

The authors have no conflicts of interest to declare. 